\title{
Relación de pareja, uso de redes sociales y mensajería instantánea en los estudiantes de la Universidad de las Islas Baleares
}

\author{
Romantic relationships and use of social networks and instant messaging among \\ students from the University of the Balearic Islands
}

\author{
Antonio Casero Martines \\ Universidad de las Islas Baleares, España, a.casero@uib.es
}

Ismael Algaba Ouled-Driss

Universidad de las Islas Baleares, España, algaba_uib@hotmail.com

Fecha de recepción: 24/06/2015, Fechas reenvíos: 8/03/2016 - 14/03/2016, 1/07/2016 -04/07/2016, 4/10/2016 - 6/10/2016. Fecha de aprobación: 13/10/2016. Fecha de publicación: 19/10/2016

\begin{abstract}
Resumen: Con el objetivo de describir el patrón de uso de dispositivos interactivos y su relación con la situación de relación de pareja, se elaboró y aplicó un cuestionario a 776 estudiantes de la Universidad de las Islas Baleares (UIB, España), mediante un procedimiento de muestreo mixto y polietápico. Por medio de pruebas de independencia, comparaciones de medias y análisis de la varianza se analizaron parámetros de uso de las redes sociales y mensajería instantánea tales como el hábito, la aplicación, y las sensaciones por deprivación, entre otros, y siempre en función de la situación de pareja y de la antigüedad de la misma. Los principales hallazgos muestran a Facebook y a WhatsApp como los medios dominantes, incluso dentro del aula, donde el aburrimiento y la necesidad de desconectar de clase son los argumentos más utilizados para justificar el uso no didáctico de los medios durante la sesión docente. También se han detectado diferencias significativas en la antigüedad de la pareja y el uso general de estos medios, y las sensaciones de malestar autoinformado por deprivación.
\end{abstract}

Palabras clave: mensajería electrónica; medios de comunicación social; estudiantes; relaciones humanas.

Abstract: In order to describe the pattern of use of interactive devices and their
connection with the status of romantic relationships, a questionnaire was prepared
and applied to 776 students from the University of the Balearic Islands (UIB, Spain),
using a mixed and multistage sampling method. By means of tests of independence,
mean comparison tests, as well as ANOVA, parameters of use on social networks
and instant messaging were analyzed, including habit, application, and feelings of
deprivation, etc., regarding the status and duration of romantic relationships. Main
findings show Facebook and WhatsApp as the most dominant apps, even in the 
classroom, where boredom and the need to disconnect from the class are the most widely used arguments to justify the non didactic use of the apps during class. Significant differences in the duration of the couple and the general use of these apps were also detected, as well as self-reported feelings of discomfort by deprivation.

Keywords: Education; national identity; teachers; patriotism; Argentina.

\section{Introducción y estado de la cuestión}

En la última década ha habido un gran desarrollo de Internet y esto ha influenciado que se creen nuevos métodos para comunicarse e intercambiar información llamados redes sociales, convertidos hoy en todo un fenómeno de masas (Flores, 2009). En la actualidad algunas de las redes sociales más utilizadas a nivel estatal son Facebook, Tuenti y Twitter (Nielsen Company, 2011). Así pues, los usuarios de Facebooky Tuenti pueden tener"amigos", como asíse denominan a los contactos de estos sitios web, con los que podrán comunicarse, compartir fotos y vídeos entre otros. En Twitter, en cambio, los contactos no se llaman "amigos", sino seguidores. Los usuarios pueden seguir la actividad de otras personas y ellos mismos pueden ser seguidos por otros usuarios. La comunicación en esta red social se lleva a cabo a través de publicaciones y comentarios; no dispone de chat.

Inicialmente, los usuarios de las redes sociales usaban como vehículo un ordenador fijo o portátil, pero con los avances tecnológicos se empezó a disponer de internet en los aparatos móviles y se creó el teléfono inteligente, difundido a nivel internacional como el Smartphone (Goacher, Goacher, Loriente \& MacHale, 2012). Los posibles usos de este tipo de teléfonos están presentes en casi todos los ámbitos, gozando de numerosas ventajas (Blanco, 2011). Así, puede verse que las nuevas tecnologías han facilitado el acceso a las redes sociales, ya que con un Smartphone lo podemos hacer desde cualquier sitio, siempre y cuando se tenga acceso a internet. Otra característica interesante de este tipo de teléfono es su mensajería instantánea, llamada WhatsApp. Este software, presente desde 2009, permite el envío de texto, imágenes, vídeo y audio, así como la localización del usuario. La diferencia entre esta aplicación para Smartphone y la mensajería móvil tradicional es que necesita conexión a internet.

Por su parte, la necesidad de afiliación, es decir, el deseo de tener amigos, establecer relaciones recíprocas o cooperar con los demás (Murray, 1938), es lo que induce a comunicarnos y expresar nuestros sentimientos con el fin de que alguien los escuche. Este intercambio de información se ha convertido en un medio muy cómodo y habitual gracias a las redes sociales y al WhatsApp.

Los universitarios utilizan las redes sociales por distintos motivos entre los cuales sabemos, por otros estudios, que son: el ocio, la vida personal, uso didáctico, etc. (Gómez, Roses \& Farias, 2012). Algunos de ellos las utilizan porque ya les venía de su etapa preuniversitaria, etapa donde las redes sociales tienen una gran influencia (Matalí \& Alda, 2009). El uso del WhatsApp juega 
un papel similar al permitir a los universitarios comunicarse con otras personas en cuestión de segundos.

Entre los jóvenes, las redes sociales se han convertido en un complemento casi imprescindible para las relaciones cara a cara para poder aumentar los niveles de confianza e intimidad con sus amigos y conocidos (Igarashi, Takai \& Yoshida, 2005), hasta el punto de que hoy día los jóvenes no son capaces de imaginarse el mundo sin los dispositivos interactivos (Buil, Roger-Loppacher \& Marimon, 2014).

En lo que se refiere a la relación de pareja, hay diversos estudios que hablan sobre las diferencias existentes entre el uso de las redes sociales por parte de personas solteras o con pareja. Quienes están solteros usan las redes sociales, entre otros, con el objetivo de encontrar pareja o un ligue esporádico (Oviedo \& Sánchez, 2005) ya que, además de lo práctico que resulta hablar con varias personas a la vez, se puede hacer desde cualquier lugar. Se hace más fácil establecer relaciones amorosas, y más simple, cuando se declaran sin pudor las necesidades que se asemejan a las propias (Búrdalo, 2000). Las personas con pareja tienen la comodidad de poder comunicarse entre ellas de una forma rápida y placentera. No obstante, las redes sociales también pueden originar un problema para las parejas cuando alguno de los miembros pasa demasiadas horas delante de la pantalla del dispositivo y no presta atención a su compañero/a. También manifiesta que esto puede favorecer el inicio de infidelidades al ofrecer un medio fácil y discretos para coquetear con otras personas, e incluso discusiones por sentirse agobiado por parte de la pareja a través de estas redes, y sentir que se tiene poca autonomía (Duran, Kelly \& Rotaru, 2011). Las distintas circunstancias pueden depender de la antigüedad de la relación, dado que la finalidad de comunicarse a través de estos medios varía conforme aumenta el tiempo de la relación. En el origen de un nexo amoroso se observa, frecuentemente dos sujetos que quieren conocer gente nueva y se encuentran, comenzando a intercambiar información sobre sus vidas para saber uno de otro, así se inicia el uso de estos medios para encontrarse, asistir juntos a eventos, etc. (Mesch, Talmud \& Quan-Haase, 2012).

Un trabajo analítico realizado por Yela (1997), puso a prueba un modelo teórico estructural del amor, fruto de la introducción de ciertas matizaciones en las cuatro dimensiones básicas: pasión erótica, pasión romántica, intimidad, y compromiso (Sternberg, 1986). La pasión erótica es aquella dimensión física-fisiológica del amor: activación general, deseo sexual, taquicardia, atracción física...etc.; la pasión romántica es aquel conjunto de ideas y actitudes vehementes sobre la pareja, propias del estereotipo del romanticismo de nuestra cultura: idealizar al otro y a la relación, creencia en algo mágico, etc.; la intimidad agrupa aspectos recíprocos relativos a un "vínculo especial de unión afectiva" (Yela, 1997), como apoyo emocional, comprensión, comunicación, confianza, autorrevelaciones, seguridad y confort junto a la pareja, etc.; el compromiso alude a decidir mantener la relación por encima de los problemas que pudiera conllevar, debido a la importancia especial conferida a la otra persona y a la propia relación. 


\section{ABra}

Vol 36, N 53, (1-15), EISSN: 2215-2997, julio-diciembre, 2016

URL: www.revistas.una.ac.cr/abra

DOI: http://dx.doi.org/10.15359/abra.36-53.2

En este trabajo, basado en una muestra de jóvenes con una media de edad de unos 22 años, el autor describe gráficamente la evolución de estas cuatro dimensiones. A partir de dicha descripción establece tres fases principales en las relaciones amorosas: una fase inicial y breve de "enamoramiento", una posterior de "amor pasional", y la más larga fase de "amor compañero". La primera fase comprende, aproximadamente, hasta el medio año de relación y se caracteriza por un aumento general de las cuatro dimensiones básicas, especialmente de la pasión erótica, la cual alcanza su punto máximo. La segunda comprende desde el medio año hasta los cuatro años aproximadamente de relación y se caracteriza por el mantener el nivel máximo de la pasión erótica y el aumento de la pasión romántica, paulatinamente. Se diferencia de la primera fase por la mayor importancia cobrada, al compararla con las otras dos, por el compromiso y la intimidad, los cuales siguen aumentando paulatinamente. La tercera fase comienza alrededor de los cuatro años y se distingue por alcanzar el nivel máximo de la intimidad y del compromiso y por el descenso paulatino de la pasión romántica y, más aún, el de la pasión erótica.

Bajo este marco de referencia el trabajo que se presenta se pretende analizar el patrón de uso de las redes sociales y del WhatsApp, por parte de los estudiantes universitarios de la Universidad de las Illes Balears (UIB), así como estudiar el posible efecto de tener pareja, y la antigüedad de la misma como tal, sobre el citado patrón de uso.

\section{Material y métodos}

\section{Participantes}

Participaron en la investigación 776 estudiantes de la Universidad de las Illes Balears (UIB), de edades comprendidas entre 18 y 67 años, con una media de 22 años y una moda de 20, resultando un error muestral para el conjunto de la muestra del $2.97 \%$, estimado para un nivel de confianza del $95 \%$ y bajo la condición más desfavorable, la de mayor varianza.

El procedimiento de muestreo fue mixto y polietápico, estratificado por estudios con selección de las unidades primarias (asignaturas) de forma aleatoria con afijación simple (una asignatura de cada estudio), y de las unidades secundarias (alumnos) mediante muestreo incidental en el aula. La proporción entre los sexos es del $54,8 \%$ en las mujeres y del $45,2 \%$ en los hombres. El $52,1 \%$ son solteros y el $47,4 \%$ tienen pareja. De los que tienen pareja, el $25,6 \%$ llevan menos de un año con ella, el 35,9\% lleva entre uno y tres años y el 38,5\% lleva más de tres años.

\section{Procedimiento}

Para llevar a cabo el proceso de recogida de datos se desarrolló un cuestionario para contestar de forma individual que se aplicó a cada uno de los grupos en sus respectivas aulas, inmediatamente antes, o después de una sesión de clase. Se solicitó sinceridad en la respuesta 
asegurando el anonimato. Con el objetivo de resolver las dudas que pudieran surgir, los encuestadores permanecieron en el aula hasta la finalización de la aplicación del cuestionario.

\section{Instrumentos}

La encuesta se conforma de los siguientes apartados:

Datos sociodemográficos y académicos. Se analizaron diferentes variables, tales como sexo, edad, si se tiene pareja y antigüedad de esta, carrera y curso actual y resultados académicos en diversas asignaturas. Parámetros básicos del uso de las redes sociales y el WhatsApp. Se analizaron descriptivos básicos como la redes sociales que se utilizan, la cantidad en que se emplean, si se tiene WhatsApp en el móvil, personas con las que se intercambian mensajes, cantidad que se envían al día, si se consultan estas nuevas tecnologías durante las clases y el motivo, etc.

Sensaciones/acciones frente al uso de las redes sociales y el WhatsApp. Se evaluó el grado conforme a algunas afirmaciones relacionadas con la interacción de los universitarios con las redes sociales. Los sujetos debían opinar: a) Qué sensaciones/acciones llevó a cabo delante $\mathrm{X}$ situación, por ejemplo: Me cuesta no contestar un mensaje cuando acabo de recibirlo, y b) Molestia causada cuando los demás llevan a cabo una acción, por ejemplo: Me molesta que mis compañeros consulten las redes sociales en el tiempo de clase. Para la obtención de dichos datos se ha usado una escala Likert de 5 opciones: $1=$ nada de acuerdo, 2=poco ce acuerdo, 3=medianamente de acuerdo, 4=bastante de acuerdo, 5=totalmente de acuerdo. La escala se aplicó a 29 ítems.

Situaciones de consulta de redes sociales/WhatsApp. Se midieron diversas situaciones en las que los sujetos consultaban el móvil, por ejemplo, mientras se está con la pareja, mientras se está hablando con otra gente... La escala utilizada fue una escala tipo Likert de cinco opciones (nunca, casi nunca, a veces, casi siempre y siempre).

Malestar por la deprivación a consultar las redes sociales y el WhatsApp (los mensajes). Se midió el nivel de malestar que sentían los sujetos ante la deprivación a poder usar sus redes sociales/WhatsApp durante un tiempo definido (10 minutos, 1 hora, 1 día, 3 días, 1 semana). La escala utilizada fue una escala tipo Likert de 11 puntos (del 0 al 10), donde $0=$ ningún malestar y $10=$ malestar insoportable.

Para la explotación de datos se utilizó el software específico SPSS (Stadistical Package for Social Sciences) v. 15.Análisis y resultados. Patrón de uso de las redes sociales y del WhatsApp 


\section{Posesión de redes sociales}

El 93,2\% de los participantes en el estudio indican que están en alguna red social. El 93\% de todos los estudiantes universitarios de sexo femenino disponen de alguna red social y el 93,3\% de los de sexo masculino, también.

\section{Red social y su uso}

La red social por excelencia entre los alumnos universitarios es Facebook, con un 92\% de usuarios. Le siguen Tuenti, con un $30 \%$ y luego Twitter con un $27 \%$.

\section{Amigos/seguidores de las redes sociales}

Facebook: La media de amigos en esta red social es de 294, con un mínimo de 0 y un máximo de 1900 amigos. Tuenti: La media de amigos en esta red social es de 154, con un mínimo de 0 y un máximo de 830 amigos. Twitter: La media de seguidores que tienen nuestros universitarios es de 58, con un mínimo de 0 y un máximo de 2000 seguidores. Y la media de personas a las que siguen nuestros universitarios es de 72 , con una mínimo de 0 y un máximo de 800 personas seguidas.

\section{Tipo de móvil y disponibilidad de WhatsApp}

El 69,5\% de los participantes en el estudio disponen de un móvil con acceso a internet (Smartphone), el 29,5\% disponen de un móvil sin internet y solo un 5\% no dispone de móvil. De los propietarios de un móvil con acceso a internet, el 67,7\% disponen de WhatsApp.

\section{Media de personas con las que se intercambian mensajes/correos en un día}

El $68 \%$ de los estudiantes afirman que se intercambian mensajes con cinco personas o menos en un día y un $25 \%$ lo hacen con entre 6 y 10 personas.

\section{Mensajes enviados/recibidos en un día}

Recibidos: Los estudiantes reciben una media de 10 mensajes o menos al día (52,9\%), más próximos a los 9-10 mensajes. Seguidamente, hay un 23,1\% que reciben entre 11 y 25.

Enviados: Los estudiantes también suelen enviar una media de 10 mensajes o menos al día $(62,6 \%)$, más próximos a los 9-10 mensajes.

\section{Personas con las que más intercambias mensajes}

Observamos que, a nivel general, los universitarios intercambian más mensajes con sus amigos, seguido de su pareja, su familia y, para terminar, sus compañeros de trabajo.

\section{Consulta de las redes sociales durante las clases y el motivo por el cual se hace}

La opción más escogida ha sido "a veces", mientras que la media se encuentra entre "casi nunca" y "a veces". El 50,2\% de los estudiantes encuestados han reconocido que consultan las redes 
sociales entre una y tres veces durante cada hora de clase. Las causas principales por la cual los alumnos consultan las redes sociales durante las clases son el aburrimiento provocado por algunas clases y la necesidad de un descanso.

\section{Resultados en relación a la pareja}

\section{Posesión de redes sociales}

Respecto al hecho de tener pareja o no, se observa que no hay diferencias significativas a la hora de estar en alguna red social. Respecto a la antigüedad de la pareja, nos referiremos a las diversas etapas de la siguiente forma: baja (menos de un año), media (entre uno y tres años) y alta (más de tres años). Podemos observar como, en el caso de Twitter, no hay diferencias significativas con la antigüedad de la pareja pero sí que se encuentran en Facebook y en Tuenti. En el caso de Facebook las parejas cuya antigüedad es baja lo utilizan un 97,9\%, un 93,9\% las de antigüedad media y un $88,2 \%$ las de antigüedad alta ( $X 2=8,617 ; \mathrm{p}=0,013)$. El Tuenti es usado un $47,4 \%$ por las parejas cuya antigüedad es baja, un 31,2\% las de antigüedad media y un $21,8 \%$ las de antigüedad alta $(X 2=16,699 ; \mathrm{p}<0,001)$.

\section{Amigos/seguidores de las redes sociales}

No se encuentran diferencias significativas en relación a tener pareja y el número de amigos/ seguidores. En lo relativo a la antigüedad de la pareja, se encuentran diferencias significativas entre los sujetos con una antigüedad baja y los que tienen una antigüedad alta con el número de amistades de Facebook ( $F=4,286 ; p=0,015)$, y Tuenti $(F=3,797 ; p=0,025)$, en donde los sujetos con una antigüedad alta tienen menos amistades que los que tienen una antigüedad baja. La media de amistades de las parejas de antigüedad baja es de 351,88 en Facebook y de 192,69 en Tuenti y la media de las parejas de antigüedad alta es de 268,5 en Facebook y 106,41 en Tuenti. No encontramos diferencias significativas con los sujetos que siguen en Twitter ni con los que son seguidos.

\section{Tipo de móvil y disponibilidad de WhatsApp (aplicación de mensajería instantánea)}

Tanto el tipo de móvil como la disponibilidad de WhatsApp son dos variables que no tienen ningún tipo de relación con el hecho de tener pareja. Tampoco se encuentran diferencias significativas entre la duración de la pareja y el tipo de móvil usado, ni con la disponibilidad de WhatsApp.

\section{Media de personas con las que se intercambian mensajes/correos en un día}

Se encuentran diferencias significativas en cuanto al hecho de tener pareja y el número de personas con las que se intercambian mensajes $(t=5,365 ; p<0,001)$. Los alumnos solteros $(1,53)$ intercambian más mensajes con distintas personas que los alumnos con pareja $(1,27)$. Sí se encuentran diferencias con la antigüedad de la pareja $(F=5,893 ; p=0,03)$, los sujetos con 
una antigüedad baja $(1,44)$ se intercambian mensajes con más personas que los que tienen relaciones altas $(1,18)$.

\section{Mensajes enviados/recibidos en un día}

No se encuentran diferencias significativas en cuanto a tener pareja y la cantidad de mensajes enviados/recibidos al día. Tampoco se encuentran diferencias significativas en relación a la duración de las parejas.

\section{Consulta de las redes sociales durante las clases y el motivo por el cual se hace}

No hay diferencias significativas en el hecho de tener pareja y consultar las redes sociales durante las clases. Se encuentran desigualdades significativas en relación a la antigüedad de la pareja y consultar las redes sociales en clase $(F=9,379 ; p<0,001)$. Las disimilitudes encontradas son entre las parejas cuya antigüedad es baja $(2,87)$ con las de antigüedad media $(2,35)$ y con las de antigüedad alta $(2,22)$. Aunque los tres grupos afirmen que lo hacen casi nunca, vemos como las consultas de las redes sociales en el aula disminuyen conforme aumenta la antigüedad de la pareja.

Algunos de los motivos por los cuales se lleva a cabo esta acción sí dependen de tener pareja. Los motivos que han aparecido son los siguientes: no entiendo la explicación $(t=2,711 ; p=0,007)$ y necesito un descanso $(t=2,012 ; p=0,045)$. Observamos que los universitarios solteros se excusan con estos argumentos en mayor proporción que los emparejados. En cambio, estos mismos motivos no parecen depender de la antigüedad de la pareja.

\section{Sensaciones/acciones frente al uso de las redes sociales y el WhatsApp}

Para llevar a cabo estas comparaciones, se han escogido aquellos ítems que son más relevantes para este estudio. La figura 1 recoge los datos según el grado de acuerdo.

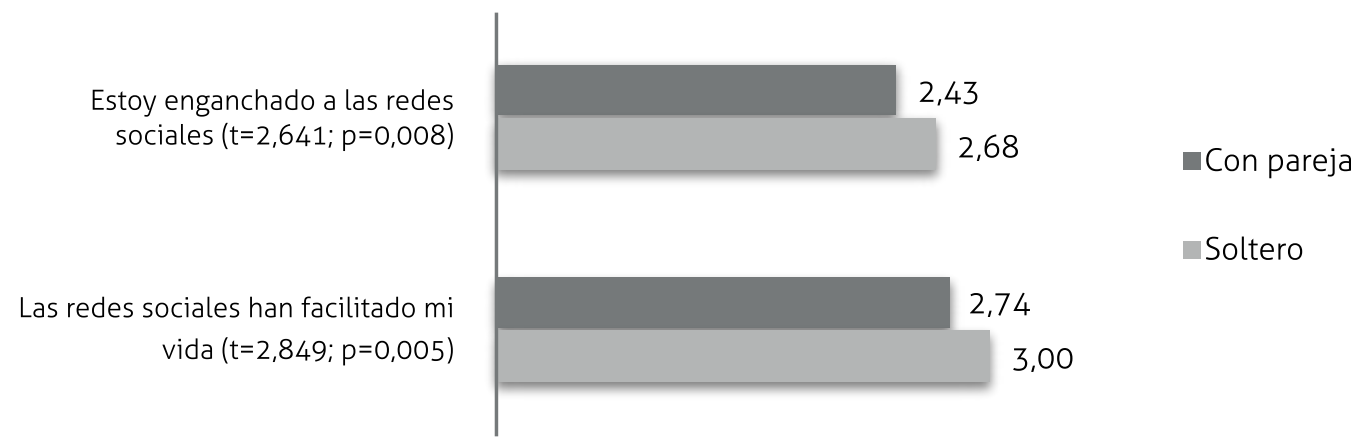

Figura 1: Grado de acuerdo con las sensaciones frente al uso de las redes sociales en función a la relación de pareja. Fuente: elaboración propia a partir de los resultados. 
En cuanto a la antigüedad de la relación en pareja, la tabla 1 muestra el resultado del análisis de la varianza de las sentencias presentadas en el cuestionario bajo cada una de las tres categorías de antigüedad de relación de pareja. Las respuestas ocupan una escala tipo Likert de 5 puntos, donde 1 indica "nada de acuerdo", y 5 indica "totalmente de acuerdo". Como puede observarse, la antigüedad de la relación provoca diferencias significativas entre las medias de las distintas sentencias, excepto en el caso del primer y tercer ítem, donde el grado de acuerdo con no poder salir de casa sin el móvil, y evitar estar en sitios con poca cobertura es independiente de la antigüedad de la relación.

Tabla 1

Sensaciones/acciones frente al uso de las redes sociales y el WhatsApp en función de la antigüedad de la pareja

\begin{tabular}{|c|c|c|c|c|c|c|}
\hline Sentencia & $\begin{array}{l}\text { Antigüedad } \\
\text { relación }\end{array}$ & Media & SD & $\mathrm{n}$ & $\mathrm{F}$ & Sig. \\
\hline \multirow{3}{*}{ No podría salir de casa sin el móvil } & Menor a 1 año & 3,11 & 1,38 & 95 & \multirow{3}{*}{0,23} & \multirow{3}{*}{ ns } \\
\hline & Entre 1 y 3 años & 3,09 & 1,42 & 134 & & \\
\hline & Mayor a 3 años & 2,99 & 1,48 & 142 & & \\
\hline \multirow{3}{*}{$\begin{array}{l}\text { Consulto si tengo mensajes nuevos } \\
\text { aunque el móvil no me haya } \\
\text { avisado }\end{array}$} & Menor a 1 año & 2,93 & 1,36 & 96 & \multirow{3}{*}{5,30} & \multirow{3}{*}{0,005} \\
\hline & Entre 1 y 3 años & 2,86 & 1,39 & 133 & & \\
\hline & Mayor a 3 años & 2,42 & 1,33 & 144 & & \\
\hline \multirow{3}{*}{$\begin{array}{l}\text { Evito estar en sitios con poca } \\
\text { cobertura }\end{array}$} & Menor a 1 año & 1,79 & 1,10 & 95 & \multirow{3}{*}{1,55} & \multirow{3}{*}{ ns } \\
\hline & Entre 1 y 3 años & 1,56 & 0,91 & 133 & & \\
\hline & Mayor a 3 años & 1,64 & 0,98 & 143 & & \\
\hline \multirow{3}{*}{$\begin{array}{l}\text { Cuando recibo un mensaje } \\
\text { necesito contestarlo enseguida }\end{array}$} & Menor a 1 año & 2,68 & 1,23 & 96 & \multirow{3}{*}{4,72} & \multirow{3}{*}{0,009} \\
\hline & Entre 1 y 3 años & 2,39 & 1,19 & 134 & & \\
\hline & Mayor a 3 años & 2,20 & 1,16 & 143 & & \\
\hline \multirow{3}{*}{$\begin{array}{l}\text { Me cuesta no contestar cuando } \\
\text { acabo de recibir un mensaje }\end{array}$} & Menor a 1 año & 2,75 & 1,34 & 96 & \multirow{3}{*}{7,13} & \multirow{3}{*}{0,001} \\
\hline & Entre 1 y 3 años & 2,57 & 1,32 & 134 & & \\
\hline & Mayor a 3 años & 2,15 & 1,19 & 143 & & \\
\hline \multirow{3}{*}{$\begin{array}{l}\text { Me preocupa no enterarme de que } \\
\text { he recibido un mensaje }\end{array}$} & Menor a 1 año & 2,25 & 1,20 & 93 & \multirow{3}{*}{4,46} & \multirow{3}{*}{0,012} \\
\hline & Entre 1 y 3 años & 2,20 & 1,08 & 133 & & \\
\hline & Mayor a 3 años & 1,88 & 1,03 & 144 & & \\
\hline \multirow{3}{*}{$\begin{array}{l}\text { Las redes sociales han facilitado } \\
\text { mi vida }\end{array}$} & Menor a 1 año & 3,15 & 1,32 & 94 & \multirow{3}{*}{8,77} & \multirow{3}{*}{$<0.001$} \\
\hline & Entre 1 y 3 años & 2,79 & 1,25 & 135 & & \\
\hline & Mayor a 3 años & 2,45 & 1,24 & 144 & & \\
\hline
\end{tabular}




\begin{tabular}{|c|c|c|c|c|c|c|}
\hline \multirow{3}{*}{$\begin{array}{l}\text { Me he sentido obligado a contestar } \\
\text { algún mensaje lo más rápido } \\
\text { posible }\end{array}$} & Menor a 1 año & 3,03 & 1,35 & 92 & \multirow{3}{*}{3,22} & \multirow{3}{*}{0,041} \\
\hline & Entre 1 y 3 años & 2,73 & 1,32 & 133 & & \\
\hline & Mayor a 3 años & 2,58 & 1,37 & 144 & & \\
\hline \multirow{3}{*}{$\begin{array}{l}\text { Estoy enganchado a la mensajería } \\
\text { electrónica (sms, WhatsApp, } \\
\text { Messenger,...) }\end{array}$} & Menor a 1 año & 2,61 & 1,46 & 95 & \multirow{3}{*}{3,81} & \multirow{3}{*}{0,023} \\
\hline & Entre 1 y 3 años & 2,35 & 1,32 & 135 & & \\
\hline & Mayor a 3 años & 2,13 & 1,20 & 144 & & \\
\hline \multirow{3}{*}{$\begin{array}{l}\text { Estoy enganchado a las redes } \\
\text { sociales }\end{array}$} & Menor a 1 año & 2,95 & 1,38 & 95 & \multirow{3}{*}{15,47} & \multirow{3}{*}{$<0.001$} \\
\hline & Entre 1 y 3 años & 2,48 & 1,28 & 135 & & \\
\hline & Mayor a 3 años & 2,03 & 1,15 & 144 & & \\
\hline
\end{tabular}

Fuente: elaboración propia a partir de los resultados.

\section{Situaciones de consulta de redes sociales/mensajería electrónica}

No se han encontrado diferencias significativas entre las consultas y el hecho de ser soltero o tener pareja. En el caso de la antigüedad de la pareja, los resultados presentan diferencias significativas respecto a "consulto el móvil cuando estoy hablando con otra gente" ( $F=4,241$; $p=0,015)$. Los universitarios con un año de relación casi nunca consultan el móvil cuando están hablando con otra gente mientras que los que llevan más de tres años de relación no lo hacen nunca.

\section{Malestar por la deprivación a las redes sociales/WhatsApp}

No se encuentran diferencias significativas entre la relación de pareja y el malestar producido. Sin embargo, sí las hay al comparar el malestar surgido de la deprivación con la antigüedad de la pareja. La tabla 2 recoge los resultados en función del grado de malestar, donde las medias indican un mayor nivel de malestar percibido a medida que aumenta el tiempo de deprivación. Mientras que la antigüedad de la relación parece producir un efecto importante entre el primer grupo (menor a 1 año) con respecto al conjunto del segundo y tercer grupo (Entre 1 y 3 años, y mayor a 3 años). Es decir, pasado el primer año de relación parece disminuir el malestar por no disponer del dispositivo, en cada uno de los 5 periodos de deprivación. 
Tabla 2

Grado de malestar según deprivación y antigüedad de la relación

\begin{tabular}{|c|c|c|c|c|c|c|}
\hline Periodo de deprivación & Antigüedad relación & Media & SD & $\mathrm{n}$ & $\mathrm{F}$ & Significación \\
\hline \multirow{3}{*}{10 minutos } & Menor a 1 año & 0,67 & 1,35 & 94 & & \\
\hline & Entre 1 y 3 años & 0,30 & 1,08 & 133 & 3,97 & 0,020 \\
\hline & Mayor a 3 años & 0,32 & 0,83 & 144 & & \\
\hline \multirow{3}{*}{ Una hora } & Menor a 1 año & 1,40 & 1,95 & 93 & & \\
\hline & Entre 1 y 3 años & 0,77 & 1,35 & 133 & 4,603 & 0,011 \\
\hline & Mayor a 3 años & 0,88 & 1,56 & 144 & & \\
\hline \multirow{3}{*}{ Un día } & Menor a 1 año & 3,48 & 2,62 & 92 & & \\
\hline & Entre 1 y 3 años & 2,86 & 2,26 & 133 & 3,293 & 0,038 \\
\hline & Mayor a 3 años & 2,64 & 2,55 & 143 & & \\
\hline \multirow{3}{*}{ Tres días } & Menor a 1 año & 5,17 & 2,81 & 93 & & \\
\hline & Entre 1 y 3 años & 4,52 & 2,61 & 133 & 3,708 & 0,025 \\
\hline & Mayor a 3 años & 4,14 & 3,09 & 144 & & \\
\hline \multirow{3}{*}{ Una semana } & Menor a 1 año & 7,00 & 2,82 & 93 & & \\
\hline & Entre 1 y 3 años & 6,23 & 2,89 & 133 & 5,336 & 0,005 \\
\hline & Mayor a 3 años & 5,65 & 3,45 & 144 & & \\
\hline
\end{tabular}

Fuente: elaboración propia a partir de los resultados.

\section{Discusión y conclusiones}

Las redes sociales son un medio deseable para los estudiantes universitarios, de hecho, el 93,2\% de los participantes en el estudio indican que están en alguna red social. La red social más presente y más utilizada, con diferencia, es Facebook con el 92\% de usuarios en esta muestra y con una media de 294 amigos. El WhatsApp es otro medio muy presente. Los universitarios se intercambian mensajes a través de estos medios con una frecuencia relativamente baja si los comparamos con los adolescentes (Echeburúa y Corral, 2010), los cuales son grandes consumidores y víctimas potenciales de adicciones tecnológicas. En el trabajo presentado se ha visto que el $68 \%$ de los estudiantes afirman que se intercambian mensajes con cinco personas o menos en un día, y un $25 \%$ que se comunican con entre 6 y 10 personas. En cuanto al número de mensajes recibidos en un día, un $52,9 \%$ de los estudiantes reciben una media de 10 mensajes o menos y un $23,1 \%$ reciben entre 11 y 25 mensajes. Los mensajes enviados son menos, ya que el dato más marcado es que el 62,6\% envían una media de 9-10 al día. Así, los universitarios de este estudio no aumentan la comunicación de estos medios, dado que las 
medias obtenidas están dentro de los niveles más bajos de la escala utilizada.

El 50,2\% de los estudiantes encuestados han reconocido que consultan las redes sociales entre una y tres veces durante cada hora de clase. Los motivos principales que se han obtenido para justificar esto han sido el aburrimiento y la necesidad de un descanso (desconectar). Este resultado es muy importante, ya que se puede observar que son muchos los alumnos que desvían su atención de las clases para entrar en el mundo virtual.

Respecto a este primer objetivo, podemos concluir en que las redes sociales y el WhatsApp son medios muy utilizados y deseados entre los universitarios por un motivo muy básico e incluso, natural: la necesidad de afiliación (Murray, 1938). Es evidente que si existe un medio por el cual se pueda saciar mejor esta necesidad, se aproveche. Respecto a la cantidad de mensajes que se intercambian al día, podemos considerar que no es tampoco muy elevada (por lo menos en nuestra muestra y teniendo en cuenta que engloba los mensajes en redes sociales y a través de WhatsApp), por lo que podemos intuir que gran parte del tiempo dedicado a las redes sociales es destinado a informarse sobre las actividades de otros usuarios, leer noticias, artículos, chistes... Y, para terminar, un aspecto que resulta preocupante: el uso de estos medios en tiempo de clase. Esta actividad se puede observar cada vez más en las aulas y esto demuestra que la innovación puede ser un "arma de doble filo" ya que, en algunos casos, podría acabar resultando contraproducente.

Respecto a las diferencias en función de tener pareja y de su antigüedad los resultados obtenidos permiten una serie de reflexiones que se consideran de interés. Aunque no se han encontrado muchas diferencias significativas en relación a tener pareja o ser soltero, se puede afirmar que, en general, los universitarios que no tienen pareja intercambian mensajes con un mayor número de personas. Esto es debido a que las personas solteras suelen utilizar, entre otros, estos medios (especialmente las redes sociales) para encontrar pareja o algún ligue gracias a la comodidad y seguridad que pueden mostrar frente a estos medios de comunicación (Búrdalo, 2000). En la misma línea se ha observado que los universitarios solteros están de acuerdo, en mayor grado que los que no lo son, con las afirmaciones "las redes sociales han facilitado mi vida" $y$ "estoy enganchado a las redes sociales". Este resultado, también, demuestra que los universitarios solteros usan de forma más asidua estos medios de comunicación al compararlos con los que tienen pareja.

Sí se han encontrado más diferencias significativas en relación a la antigüedad de la pareja. Concretamente, las diferencias más notables han sido entre las relaciones bajas y las altas. Así pues, se observa que las redes sociales Facebook y Tuenti están más presentes y son más usadas por las parejas de antigüedad baja. De la misma manera, estos intercambian mensajes con más personas que los que tienen una antigüedad es alta. Cuando la antigüedad es baja también intercambian más mensajes durante las clases que las parejas cuya antigüedad es alta. 


\section{ABra}

Vol 36, N 53, (1-15), EISSN: 2215-2997, julio-diciembre, 2016

URL: www.revistas.una.ac.cr/abra

DOI: http://dx.doi.org/10.15359/abra.36-53.2

Respecto a las sensaciones/acciones frente al uso de las redes sociales y el WhatsApp, se han encontrado muchas diferencias significativas cuando se comparan los distintos tiempos de la duración. En este caso se ha podido observar que el "enganche" a las redes sociales y al WhatsApp no solo continúa estando muy presente en las relaciones bajas, sino que también se encuentra latente en las relaciones medias. Podemos ver que la necesidad a la hora de contestar un mensaje, el tiempo que se tarda en hacerlo y estar pendiente de haberlo recibido así como reconocer que se está enganchado a las redes sociales, al WhatsApp o pensar que las redes sociales han facilitado sus vidas son sensaciones que están muy presentes durante el comienzo de la relación (el primer año) y van decreciendo a medida que pasa el tiempo (entre el primer y el tercer año todavía podemos encontrar algunas que son bastante latentes) hasta prácticamente desaparecer cuando la antigüedad es alta. En situaciones de consulta de las redes sociales y el WhatsApp observamos una tendencia a consultar estos medios mientras se está hablando con otra gente. Esta tendencia, presente en parejas cuya antigüedad es baja e inexistente en antigüedades altas, es a causa del "enganche" que se tiene a estos medios. Otra característica de este "enganche" es el malestar que presentan las parejas de antigüedad baja frente a la deprivación de las redes sociales y el WhatsApp durante un intervalo de tiempo que abarca de los diez minutos hasta una semana. A medida que pasa el tiempo sin poder consultar los mensajes aumenta el grado de malestar que presentan los universitarios, especialmente, los que tienen una antigüedad baja, los cuales presentan unos grados de malestar muy superiores a los que tienen una antigüedad alta.

Como hemos podido ver, las redes sociales y el WhatsApp son recursos que están presentes en todos los universitarios independientemente de su condición de pareja. No obstante, el uso que se tiene de estos recursos varía en función de ser soltero o no y, sobre todo, según la antigüedad de la pareja. Es aquí donde el modelo de Yela (1997) puede facilitar la comprensión de los hallazgos; según este, en la primera fase, llamada "enamoramiento", la pasión erótica y la pasión romántica juegan un papel protagonista en las relaciones, estas dos dimensiones pasionales, presentes en los universitarios con parejas cuya antigüedad es baja, se encargan de que la relación tenga interés por los dos miembros y siga adelante. En este punto, el compromiso no está muy presente (pero empieza a aumentar) y es por este motivo que los que tienen una antigüedad de pareja baja todavía disponen de cierta libertad. Pasado el primer medio año de la relación, se entra en la fase de "amor pasional", la cual se extenderá hasta los cuatro años. En este periodo, que englobaría a los universitarios con una antigüedad media y una pequeña proporción de los que tienen una antigüedad alta, se encuentra que el compromiso empieza a aumentar y es este factor el que hace que la relación comience a ser estable (con ayuda de la intimidad) y, consecuentemente, provoca que las parejas cambien sus hábitos antiguos como podría ser el enganche a las redes sociales, comunicarse con varias personas, etc. La presión que se pueda originar a través de estos problemas no llega a ser lo suficientemente fuerte como para romper la pareja porque se encuentran en el mayor grado de la pasión erótica (alcanzado en la primera fase), y una importante presencia de la pasión romántica, las cuales 
hacen que se sientan muy atraídos entre ellos y acaben adaptándose a las nuevas circunstancias, al nuevo estilo de vida. Por último, se encuentra la fase de "amor compañero". En ella, la cual empieza a partir de los cuatro años y se mantiene a lo largo de la relación, las dimensiones pasionales empiezan a disminuir mientras que el compromiso y la intimidad alcanzan el mayor grado. Esta fase se puede identificar con la producida en antigüedades altas de relación, presente en este estudio donde se puede observar como los universitarios que tienen este perfil han dejado completamente de lado sus antiguos hábitos con estas nuevas tecnologías y se han acomodado en esta incipiente fase, la cual, no es muy compatible con el uso que se daba antes a estos recursos.

\section{Referencias bibliográficas}

Blanco, S. (2011). No sin mi SmartPhone. Uciencia, 6, 48-50.

Buil, P., Roger-Loppacher, O. \& Marimon, F. (2014) The impact of SMS messages on young people's participation in recycling campaigns, Communication \& Society / Comunicación y Sociedad, Vol. 27, n. 1, p. 161-182.

Búrdalo, B. (2000). Amory Sexo en Internet. Madrid, Biblioteca Nueva.

Duran, R. L., Kelly, L., \& Rotaru, T. (2011). Mobile Phones in Romantic Relationships and the Dialectic of Autonomy Versus Connection. Communication Quarterly, 59 (1), 19-36.

Echeburúa, E.C, y de Corral, P. (2010). Adicción a las nuevas tecnologías y a las redes sociales en jóvenes: Un nuevo reto. Adicciones, vol. 22, n², 91-96.

Flores, J.M. (2009): Nuevos modelos de comunicación, perfiles y tendencias en las redes sociales, Comunicar, 33, 73-81. (DOI: 10.3916/c33-2009-02-007).

Goacher, A., Goacher J., Loriente J. y MacHale E. (2012). El uso de smartphones en España. Recuperado de www.go-movil.es/index.php/el-uso-de-smartphones-en-espana-febrero-2012/.

Gómez, M., Roses, S., y Farias, P. (2012). El uso académico de las redes sociales en universitarios. Comunicar, 38, 131-138. (DOI: 10.3916/C38-2011-03-04).

Igarashi, T., Takai, J.\&Yoshida, T. (2005). Gender differences in social network development via mobile phone text messages: A longitudinal study. Journal of Social and Personal Relationships, 22, 691-713. 
Matalí, J. L., y Alda, J. Á. (2009). Adolescentes y nuevas tecnologías: ¿innovación o adicción?Barcelona, Edebé.

Mesch, G., Talmud, I., \& Quan-Haase, A. (2012). Instant messaging social networks: Individual, relational, and cultural characteristics. Journal of Social and Personal Relationships, 1-24.

Murray, H. (1938). Exploración de la personalidad. USA, Mc.Graw Hill.

Nielsen Company (2011). The social media view from Spain. (2012). Recuperado de http://www. slideshare.net/retelur/the-social-media-view-from-spain-nielsen-nov11.

Oviedo, L., y Sánchez, J. (2005). Amor.com: Vínculos de pareja por internet. Revista Intercontinental de Psicología y Educación, 43-56.

Sternberg, R. (1988). El Triángulo del Amor: Intimidad, amor, compromiso. Barcelona, Paidós.

Yela, C. (1997). Curso temporal de los componentes básicos del amor a lo largo de la relación de pareja. Psicothema. Vol. 9, n 1, 1-15. 